\title{
ZFAS
}

\section{Einleitung: „Neue“ deutsche Außenpolitik?}

\author{
Gunther Hellmann • Daniel Jacobi • Ursula Stark Urrestarazu
}

(C) Springer Fachmedien Wiesbaden 2014

Die Rede von einer „neuen deutschen Außenpolitik“ ist im Jahr 2014 fast zu einer stehenden Formulierung geworden. Eine schnelle Recherche im Internet belegt dies eindrucksvoll. Nun hat es Phasen wachsenden Interesses an deutscher Außenpolitik im Verlauf der letzten Jahrzehnte immer wieder gegeben. Dies gilt insbesondere für die Zeit unmittelbar nach der deutschen Vereinigung und nach dem Regierungswechsel von Helmut Kohl zu Gerhard Schröder 1998. Selten aber war der Beginn einer neuen und intensiven Diskussion über den Standort und die Perspektiven deutscher Außenpolitik so eindeutig datierbar wie im Fall der gegenwärtigen laufenden Debatte, die mit den Reden von Bundespräsident Gauck (Gauck 2014) und Außenminister Steinmeier (Steinmeier 2014a) bei der Münchner Sicherheitskonferenz 2014 ihren Anfang nahm. Und selten wurde der neue Anspruch ambitionierter formuliert.

Bemerkenswert ist auch, dass es im Kontrast zu vorangehenden Diskussionen über eine „,neue deutsche Außenpolitik“ keinen unmittelbaren externen Anlass für eine solche Diskussion gab - wie etwa im Sinne einer „Neuordnung“ der Beziehungen der Bundesrepublik zu den drei Westmächten im Zuge der Wiederbewaffnung in den 1950er Jahren (Haftendorn 2001, S. 33-38); der ,neuen Ostpolitik“ in den 1970er Jahren als Reaktion auf und in Ergänzung zur westlichen Entspannungspolitik (Bender 1986, S. 115-137); der deutschen Vereinigung 1990, die aus naheliegenden Gründen Anlass zur bislang ausgeprägtesten Debatte über eine ,neue deutsche

G. Hellmann $(\bowtie) \cdot$ D. Jacobi · U. Stark Urrestarazu

Fachbereich 03: Institut für Politikwissenschaft, Goethe Universität Frankfurt,

Campus Westend, PEG-Gebäude; Fach 24, Grüneburgplatz 1,

60323 Frankfurt am Main, Deutschland

E-Mail: g.hellmann@soz.uni-frankfurt.de

D. Jacobi

E-Mail: jacobi@soz.uni-frankfurt.de

U. Stark Urrestarazu

E-Mail: stark@soz.uni-frankfurt.de 
Außenpolitik“ gab; ${ }^{1}$ dem Regierungswechsel von Schwarz-Gelb zu Rot-Grün 1998 als der neue Bundeskanzler Gerhard Schröder ,das Selbstbewusstsein einer erwachsenen Nation“ in den Mittelpunkt seiner ersten Regierungserklärung rückte (Hellmann 1999); oder den Anschlägen des 11. September 2001 und des nachfolgenden Zerwürfnisses zwischen den USA und Deutschland über einen Angriff auf Saddam Husseins Irak im Frühjahr 2003 (Schöllgen 2003; Risse 2004). Gewiss, die Sicherheitskonferenz tagte nur wenige Wochen nach der Aufnahme der Regierungsgeschäfte durch die neue Koalition zwischen CDU/CSU und SPD. Aber da mit Frank-Walter Steinmeier ein alter Bekannter auf den Platz des Außenministers zurückkehrte, der bereits in derselben Konstellation mit der Bundeskanzlerin vier Jahre regiert hatte und weder die Koalitionsvereinbarung noch die ersten Regierungserklärungen Hinweise auf größere Einschnitte lieferten, ließ sich alleine aus dem Regierungswechsel kein Einschnitt in der deutschen Außenpolitik ableiten. Außenminister Steinmeiers Warnung vor dem ,halbstarken Gerede über wachsenden deutschen Einfluss und neue deutsche Macht“ sowie „Mittelmachtsphantasien nach dem Motto ,Wir sind wieder wer“" schienen dies zu bestätigen - trotz aller „Fragezeichen“, die er explizit dem „Gemeinplatz“ in Antrittsreden von neuen Außenministern hinzufügen wollte, lediglich „die Kontinuität zu beschwören“ (Steinmeier 2013).

Nicht weitreichende weltpolitische Veränderungen lieferten daher Anfang 2014 den Anlass für den Beginn einer der intensivsten Debatten über deutsche Außenpolitik seit dem Bestehen der Bundesrepublik, sondern das sich wechselseitig ergänzende Bestreben des Bundespräsidenten und des gerade wieder ins Amt zurückgekehrten Außenministers. Gauck und Steinmeier stimmten ihre Reden bei der international bedeutendsten Konferenz zur Sicherheitspolitik ganz bewusst auf engste miteinander $\mathrm{ab}$, um ein größtmögliches Maß an Aufmerksamkeit für einen neuen und weiterreichenden außenpolitischen Gestaltungsanspruch zu erlangen. Zudem ging es für Außenminister Steinmeier und sein Team im Auswärtigen Amt auch darum, einen wahrgenommenen Reputationsverlust des Außenministeriums während der Amtszeit von Außenminister Westerwelle rückgängig zu machen und den Einfluss des Außenministeriums in der Regierung insgesamt zu stärken (Bittner und Nass 2014; Rinke 2014). Diesem Zweck diente (und dient) auch der bereits im Dezember 2013 von Außenminister Steinmeier angekündigte ,Review 2014““-Prozess einer „Selbstverständigung über die Perspektiven deutscher Außenpolitik“, der bewusst ,als Dialog des Auswärtigen Amtes mit den wichtigsten außen- und sicherheitspolitischen Stakeholdern unter Einschluss der Zivilgesellschaft" angelegt wurde (vgl. Steinmeier 2013; 2014b sowie den Beitrag von Christoph Bertram in diesem Band).

\section{Zur Kategorie des Neuen}

Dass es seit Beginn des Jahres 2014 eine neue und ausgesprochen intensiv geführte Debatte über die deutsche Außenpolitik gibt, dürfte mittlerweile unstrittig sein. Dies

\footnotetext{
${ }^{1}$ Vgl. u. a. Maull (1992), Bredow und Jäger (1993), Hellmann (1997) sowie die vierbändige Reihe der Deutschen Gesellschaft für Auswärtige Politik unter dem Titel „Deutschlands neue Außenpolitik“ (Kaiser und Maull 1994; 1995; Kaiser und Krause 1996; Eberwein und Kaiser 1998).
} 
belegt auch dieser Band. Inwieweit diese Debatte allerdings auch eine neue deutsche Außenpolitik zum Gegenstand hat, ist hingegen stärker umstritten. Denn neben der inhaltlichen Bestimmung möglicher Veränderungen in der Ausrichtung deutscher Außenpolitik stellt sich natürlich zunächst die grundsätzliche Frage, was man unter dem Begriff des „Neuen“ verstehen soll. Hierzu kann man grundlegend zwei verschiedene Dimensionen unterscheiden, eine historische und eine analytische.

Mit einem lange in die Geschichte zurückreichenden historischen Blick spielt das Neue weder in der klassischen griechischen Philosophie noch in Texten der scholastischen Tradition eine besondere Rolle. Erst mit der Überwindung letzterer entsteht ein Interesse an der Beschreibung von „Neuem“. Die Renaissance lässt sich als der Scheitelpunkt benennen, an dem die Kategorie des Neuen erstmals reflexiv wird. In ihr wird ein Verständnis der eigenen Zeit als ,neu“ Teil der ,grundlegenden Orientierungs- und Selbstverständigungsleistungen des europäischen Denkens“" (Rath 1984, S. 727). Das Neue wird als eine Eigenart geschichtlicher Zukunft erkannt und das Interesse an ihr führt zu der Ausbildung der Wissenschaften sowie der Forderung an die Kunst, Neues zu schaffen. Die Entdeckung des amerikanischen Kontinents befördert dann auch geographisch die Differenzerfahrung von „Neuer Welt“ und „Alter Welt". Allgemein sowie insbesondere politisch gilt die Französische Revolution dann gemeinhin als die Visualisierung und/oder Kulmination des Neuen. Formal wird das Neue vor allem entlang der Unterscheidung neu/alt bis in die Gegenwart diskutiert. Dabei variiert dessen positive bzw. negative Bewertung historisch. Während das Neue vor allem in Folge der Neuzeit enthusiastisch begrüßt wurde, wird es heute tendenziell kritisch betrachtet. Insbesondere wird die grundsätzliche Möglichkeit des Neuen in vielen gesellschaftlichen Kontexten hinterfragt. Man denke nur an die Diskussion um die nichtabsehbaren Folgen technischer Neuentwicklungen.

Auch im Kontext der deutschen Außenpolitik ist die zögerliche Bezugnahme auf „Neues“ zu beobachten, insbesondere unter Praktikern. Sowohl den professionellen Beobachtern wie auch den außenpolitischen Eliten fehlte lange Zeit eine starke historische Kontrastfolie, gegen die eine ,neue“ deutsche Außenpolitik positiv konturiert werden konnte. Die Bonner Republik hatte vor allem eine klare Vorstellung davon, was sie vermeiden wollte. „Nie wieder Weimar" war anfangs ihr Credo. Dies zu erreichen, fiel ihr auch vergleichsweise leicht, wie der Schweizer Publizist Fritz René Allemann in seinem vielzitierten Buch „Bonn ist nicht Weimar“ bereits Mitte der 1950er Jahre zeigte (Allemann 1956). Eine „,selbstbewusste“ Berliner Republik hatte es demgegenüber deutlich schwerer, im Blick auf die deutsche Vergangenheit eine neue Außenpolitik positiv abzuheben, da sich mit „Selbstbewusstsein“ nicht selten solche außenpolitischen Assoziationen verbinden, die im deutschen Fall Erinnerungen an die „Machtstaatstradition“ Bismarcks oder gar Hitlers wecken. Zum anderen konnte „Bonn“ (im Unterschied zu „Weimar“) lange Zeit nicht ganz so einfach als Negativfolie dienen. Dies hat sich (wie in diesem Band an verschiedenen Stellen zu sehen ist) erst in den letzten Jahren verändert, etwa im Hinblick auf die Umdeutung der früher durchgängig positiv besetzten „Kultur der Zurückhaltung“. So dient die deutsche Enthaltung im Fall des Libyen-Mandats des UN-Sicherheitsrates mittlerweile vielen als ein prominentes Beispiel zur Illustration der vermeintlichen Schattenseiten eines solchen Verständnisses. 
In analytischer Hinsicht lässt sich feststellen, dass die Kategorie des Neuen selbst eine historische ist. Abstrakt gesprochen liefert sie eine Form der (Selbst-)Beschreibung (von Gesellschaft) und verleiht der Erfahrung einer Differenz Ausdruck, die sich aus einer Nichtvereinbarkeit einer Beobachtung mit einem alten Begriff ergibt. Insofern ist sie auch Ausdruck einer begrifflichen Hilflosigkeit, wenigstens aber erfüllt sie die Funktion eines semantischen Platzhalters. Wo Veränderungen sich andeuten oder abzeichnen, aber noch nicht ,auf den Begriff“ gebracht werden können, dient das „Neue“ in seinen unterschiedlichen Variationen als eine Übergangsbegrifflichkeit, bis diese Lücke geschlossen werden kann. Dies zeigt sich etwa beispielhaft darin, dass die Kategorie des Neuen oft in Verbindung mit Revolutionen oder der Formulierung von Utopien auftaucht.

\section{Implikationen für diesen Sammelband}

Vor allem aus dem letzten Befund lässt sich somit auch eine Begründung für die Zusammenstellung des vorliegenden Sammelbandes formulieren. Dort, wo sich Außenpolitik in einer Phase der Neu(er)findung befindet, kann die Reflexion auf das Spezifische des Neuen nicht früh genug einsetzen. Die Beteiligten müssen sich auf die Suche nach einem Vokabular machen, das die beobachtbaren Entwicklungen fassbar machen und idealiter zur Verständigung zwischen den eingebundenen Politikern, Experten und der breiteren Öffentlichkeit beitragen soll. Dies wird sich ohne kontroverse Auseinandersetzungen hier und da wohl nicht erzielen lassen. Aber Debattenbeiträge wie die in diesem Band versammelten sollen genau diese Auseinandersetzung ermöglichen. Angelegt ist dieser Band ganz bewusst als Dialog zwischen „Theorie“ und „Praxis“ der Außenpolitik. Wissenschaftler in Universitäten und Think Tanks kommen ebenso zu Wort wie Praktiker aus dem Bundestag sowie dem exekutiven Bereich. Die Beleuchtung ,alter“ wie auch potenziell ,neuer“ Facetten deutscher Außenpolitik wird (jeweils im Plural!) sowohl aus deutschen Binnenperspektiven wie auch aus der Perspektive wichtiger Partner Deutschlands vorgenommen. All dies, so die Hoffnung, soll zur Schaffung eines gemeinsamen Übersetzungsrahmens beitragen und somit der Verständigung über die sich entwickelnde Außenpolitik Deutschlands dienen.

Dies grenzt den Sammelband gleichzeitig auch von thematisch ähnlich ausgerichteten Publikationen der letzten Jahre ab. Gerade weil es zwischenzeitlich so schien, dass das „Neue“ an deutscher Außenpolitik in klarere Formen zu gerinnen schien, finden sich für die letzten Jahre bereits diverse Sammelbände, die eine Bestandsaufnahme in längerer historischer Perspektive wagen (Schmidt et al. 2007; Jäger et al. 2011). Der vorliegende Band macht es sich demgegenüber vor allem zur Aufgabe, den Ist-Zustand bzw. die Diagnose eines (möglichen) Wandels deutscher Außenpolitik aus unterschiedlichen sachlichen und professionellen Blickwinkeln aufzugreifen und die Implikationen zu analysieren, die mit potentiellen Veränderungen verbunden sein könnten. Damit greift er thematisch weiter aus als ähnliche Schwerpunkte zur 
deutschen Außenpolitik, die sich im vergangenen Jahr in anderen Fachzeitschriften fanden. ${ }^{2}$

\section{Struktur des Bandes}

Im Sinne dieser Aufgabe ist der vorliegende Band in insgesamt sechs Abschnitte gegliedert, die sich dem äußerst komplexen Themenfeld aus unterschiedlichen sachlichen und professionellen Perspektiven nähern. Denn so einfach die Thematik der (,neuen“) deutschen Außenpolitik auf den ersten Blick erscheinen mag, so komplex sind eigentlich die tieferliegenden Dimensionen des Begriffes wie auch der politischen Praxis der Außenpolitik.

Der erste Abschnitt ist der Geschichte und Gegenwart der Praxis wie auch des Begriffes der Außenpolitik gewidmet. Sowohl die historische Dimensionen des Begriffes als auch die weltpolitischen Entstehungsbedingungen dieser Praxis werden darin aus unterschiedlichen disziplinären Kontexten heraus aufgegriffen. Andreas Fahrmeir diskutiert in seinem Beitrag die Problematik gängiger staatszentrierter Verständnisse von Außenpolitik in historischer Perspektive und schlägt anhand einiger Beispiele vor, Außenpolitik im Wesentlichen als eine Praxis der Grenzziehung zwischen politischen Gemeinschaften und Politikbereichen zu denken. Matthias Albert betrachtet Außenpolitik im Kontext der Entstehung des modernen weltpolitischen Systems und beschäftigt sich auf der Grundlage differenzierungs- und systemtheoretischer Überlegungen mit den grundsätzlichen Beschränkungen der Planung von Außenpolitik. Karl Kaiser beleuchtet die strukturellen Bedingungen deutscher Außenpolitik in einer globalisierten Welt sowie damit zusammenhängende Chancen und Risiken der globalen Interdependenz. Friedrich Kratochwils Beitrag analysiert deutsche Außenpolitik im Kontext einer Krise westlicher Außenpolitik insgesamt, die als Kulminationspunkt diverser Veränderungen beschrieben werden, darunter insbesondere einer von den USA und der EU propagierten universalen „Wertepolitik“, die neben anderen Entwicklungen dazu beigetragen habe, die Spielräume verantwortlicher politischer Entscheidung zu untergraben.

Im zweiten Abschnitt steht die Rolle der Gesellschaft bzw. Öffentlichkeit(en) von Außenpolitik im Vordergrund. Was bzw. wer genau damit gemeint (oder eben nicht gemeint) ist, damit befasst sich der Beitrag von Daniel Jacobi, der die Rolle und Erreichbarkeit der Gesellschaft in der Formulierung und Rechtfertigung von Außenpolitik in den Blick nimmt. Christoph Bertram diskutiert in seinem Beitrag die Grundidee und die erhofften Resultate des von Außenminister Steinmeier mit dem „Review 2014“ angestoßenen gesellschaftlichen „Selbstverständigungsprozesses über die Perspektiven deutscher Außenpolitik. Christian Tuschhoffs Beitrag problematisiert die Rolle von Medien und Denkfabriken bei der gesellschaftlichen Debatte um deutsche Außenpolitik im Allgemeinen sowie bei der Verarbeitung der Rede von Bundespräsident Gauck im Besonderen und diskutiert die Frage, inwiefern

\footnotetext{
${ }^{2}$ Siehe etwa den Thementeil „Deutsche Außenpolitik kontrovers“ in der Zeitschrift WeltTrends Heft 96 (Mai/Juni 2014), S. 33-75 sowie den Schwerpunkt zur deutschen Außenpolitik in Heft 4 der Zeitschrift „Internationale Politik“ vom Juli/August 2014.
} 
diese Institutionen zu einer gesellschaftlich breiten Debatte um Parameter deutscher Außenpolitik beitragen.

Der dritte Abschnitt behandelt den Themenkomplex des „Neuen“ in der Außenpolitik sowohl in praktischer wie theoretischer Perspektive. Aus der Perspektive der Außenpolitikforschung diskutiert Wilfried von Bredow am Beispiel der deutschen Außenpolitik, wie ,neue“ Wahrnehmungen und Prioritäten in der Außenpolitik analysiert werden können. Ulrich Schlie zeichnet den politischen Prozess der Entstehung eines Weißbuches nach und diskutiert die Rolle eines solchen Prozesses für die Formalisierung außen- und sicherheitspolitischer Debatten. Die Herausforderungen bei der Konzeption und Umsetzung einer kohärenten Politikstrategie verdeutlicht Klaus Naumann in seinem Beitrag am Konzept der (,vernetzten“) Sicherheit und diagnostiziert dabei erheblichen Nachholbedarf bei den Strategieprozessen sowie der Außenkommunikation. Ursula Stark Urrestarazu beleuchtet schließlich die Debatte um Deutschlands „Rolle in der Welt“ aus identitätstheoretischer Perspektive und diskutiert die Frage, inwiefern die seit der Münchner Sicherheitskonferenz entflammten Debatten von einem außenpolitischen Identitätswandel zeugen. Sie argumentiert dabei, dass Identität grundsätzlich handlungs- und situationsbezogen zu verstehen ist und somit immer sowohl ,neue“ als auch ,alte“ Elemente aufzeigt.

Die beiden darauf folgenden Abschnitte greifen stärker inhaltliche Dimensionen der Debatte auf - einerseits wesentliche inhaltlich-konzeptuelle Dimensionen sowie andererseits die Dimension der Instrumente und Politikmittel („,neuer") deutscher Außenpolitik. Im vierten Abschnitt werden zunächst drei begriffliche Spannungsfelder aufgegriffen, die sich an zentralen Begriffspaaren der Debatte orientieren. Diese Begriffspaare werden jeweils von einem „Praktiker“ und einem „Akademiker“ bearbeitet, um sowohl einen distanziert-akademischen wie auch einen praxisnahen Blick auf bestimmende (und spannungsgeladene) Konzepte deutscher Außenpolitik zu ermöglichen. Welche Funktionen diesen Konzepten in der gesellschaftlichen, wissenschaftlichen und praktischen Perspektive Debatte zukommen, zeigt sich hier in voller Breite mit teils sehr unterschiedlichen Akzentuierungen. „Verantwortung und Macht" steht im Mittelpunkt der Beiträge von Joscha Schmierer und Hanns Maull. Deutsche Verantwortung in der Außenpolitik ist - so Schmierer in seinem Beitrag entgegen den gegenwärtigen Diskussionstrends nicht durch „,neue Macht“, sondern angesichts der aktuellen Gefährdungen der globalen Ordnung im Gegenteil durch „neue Ohnmacht“ geprägt. Auch Hanns Maull diagnostiziert in seinem Beitrag eine zunehmende Erosion der Verantwortungsbereitschaft wie auch der Leistungs-, also Gestaltungsfähigkeit der deutschen Außenpolitik und somit ihrer Macht.

Matthias Zimmer und Benjamin Herborth widmen sich jeweils dem Spannungsfeld „Interessen und Werte“. Letztere sind nach Matthias Zimmers Auffassung in der Außenpolitik nur dann sinnvolle Richtschnuren, wenn sie sich in Interessen umformulieren und zur Wahrung einer außenpolitisch konsistenten Strategie priorisieren lassen. Benjamin Herborth betont hingegen den Charakter von Werten und Interessen als politische Rechtfertigungsfiguren, die darauf hinauslaufen, außenpolitische Positionen aus dem Wechselspiel von Position und Opposition herauszulösen und außenpolitische Handlungen zu rechtfertigen.

Das Spannungsfeld „Führung und Zurückhaltung“steht in den Beiträgen von Rolf Mützenich und Werner Link im Zentrum. Mützenich argumentiert, dass deutsche 
Führung nur eingebettet in europäische Institutionen möglich sei. Die Debatte über Führung kranke allerdings an begrifflichen Uneindeutigkeiten, die überwunden werden müssten, um nicht falsche Erwartungen bei Deutschlands Partnern zu schüren. Ähnlich argumentiert auch der Beitrag von Werner Link, in dem die These vertreten wird, dass das Problem der Führung in der internationalen Politik eigentlich nur in strukturellen Zusammenhängen, also im Kontext der globalen Machtverteilung und deren Veränderungen, sinnvoll diskutiert werden kann und sich Deutschlands Rolle im europäischen Kontext nach wie vor in erster Linie über gemeinsame Führung definiere.

Der fünfte Abschnitt folgt einer ähnlichen Logik, die im Hinblick auf die Instrumente bzw. praktischen Politikmittel (,neuer“) deutscher Außenpolitik natürlich besonders nahe liegt. So bedeutsam die militärische Dimension ohne Frage ist - vor dem Hintergrund der ausgeprägt sicherheitspolitischen Ausrichtung der bisherigen Debatte liegt der Auswahl dieser „Instrumente“ nicht zuletzt auch die Überlegung zugrunde, die zivilen Dimensionen (,neuer“) deutscher Außenpolitik verstärkt und systematisch in den Blick zu nehmen.

Das Instrument der Diplomatie wird aus der praktischen Perspektive von Wolfgang Ischinger und Tobias Bunde beleuchtet, mit Harald Müller als wissenschaftlichem Counterpart. Beide Beiträge charakterisieren die deutsche Diplomatie als im Großen und Ganzen gut aufgestellt, Ischinger und Bunde betonen jedoch die Notwendigkeit, dieses bewährte Instrument deutscher Außenpolitik an aktuelle weltpolitische Herausforderungen anzupassen. Harald Müller warnt zudem vor der potentiellen „Selbst-Illusionierung“ deutscher Diplomatie, die durch die suggestive Kraft fest verankerter deutscher Weltbilder und Zielsysteme zu außenpolitischen „Placebos“ verleiten mag.

Roderich Kiesewetters Beitrag diskutiert die Rolle des Militärs als Praktiker bzw. Parlamentarier, Sebastian Enskat und Carlo Masala steuern die akademische Perspektive bei. In beiden Beiträgen steht der profunde Wandel der Bundeswehr als außenpolitisches Instrument im Mittelpunkt. Enskat und Masala zeichnen ihren Wandel von der Abschreckungs- zur Einsatzarmee seit dem Ende des Kalten Krieges nach und zeigen wie die Beteiligung Deutschlands an internationalen Militäreinsätzen sukzessive ausgeweitet wurde. Roderich Kiesewetter plädiert vor diesem Hintergrund in seinem Beitrag für ein grundsätzlich neues Verständnis des Militärischen im Rahmen des Konzeptes der vernetzten Sicherheit. Die Entwicklungszusammenarbeit steht hingegen im Zentrum des Beitrages von Dirk Messner. Bei allen Herausforderungen, die die weltpolitische Lage der deutschen Außenpolitik stellt, betont er in seinem Beitrag die Chance, neue Kooperationsmuster durch Transformationsallianzen mit aufsteigenden Ländern zu etablieren. Dies impliziere ein großes Transformationspotential Deutschlands für die globale Nachhaltigkeitspolitik.

Im letzten Abschnitt kommen renommierte ausländische Beobachter deutscher Außenpolitik zu Wort. Anne-Marie LeGloannec analysiert aus französischer Perspektive alte und neue Differenzen sowie die Gefahr einer potentiellen Entkopplung zwischen Deutschland und Frankreich (,decrochage“) in einer schwierigen Phase der Europäischen Union. Die deutsche Ostpolitik wird von Adam Krzemiński einem historisch informierten wie kritischen Blick unterzogen. Aus polnischer Perspektive werden insbesondere in der Ukraine-Krise - mit den Emanzipationsbestrebungen in 
Russlands „,nahem Ausland“ und dem Gewaltgebahren einer „lädierten“ Großmacht - die alten Dilemmata der 80er Jahre wieder erkennbar. Auch in Andrei Zagorskis Beitrag kommt zum Ausdruck, wie sehr die Ukraine-Krise die Wahrnehmung deutscher Außenpolitik im Ausland prägt. Da sich in Russland durch die Krise in erster Linie die realpolitisch-realistische Perspektive als dominierende Sichtweise durchgesetzt habe, sähen sich in Russland insbesondere jene Kritiker bestätigt, die Deutschland im Fahrwasser US-amerikanischer Außenpolitik sehen.

Stephen Szabo diskutiert in seinem Beitrag insbesondere die Erwartungen an mehr deutsche Führungsverantwortung aus US-amerikanischer Perspektive und die Aussicht, dass die jüngst in der Ukraine-Krise eingenommene strategische Rolle Deutschlands dennoch nicht unbedingt Washingtons Präferenzen entsprechen kann. Die gleichzeitig skeptische wie auch positive britische Reaktion auf eine führungsstärkere deutsche Rolle, die sich zu einem Zeitpunkt ereigne, an dem Großbritannien seines Großmachtstatus müde geworden ist, wird von Willie Paterson in seinem Beitrag diskutiert. Moshe Zimmermanns Beitrag beleuchtet hingegen die ,besondere Verantwortung“ Deutschlands gegenüber Israel, und argumentiert, dass im Rahmen einer ,neuen“ deutschen Außenpolitik der historische Zusammenhang zwischen dem Staat Israel und der Shoa in neuer Form in die Einstellung zum Nahen Osten eingeordnet werden müsse.

Dieser Blick ,von außen“ liefert insbesondere im Hinblick auf die vieldiskutierte Dimension der „Erwartung“ internationaler Partner an die deutsche Außenpolitik interessante Überlegungen und zeichnet auch hier ein sehr facettenreiches und instruktives Bild ausländischer Wahrnehmungen der Debatte. Durch die sowohl praktischen als auch wissenschaftlichen Perspektiven, die zudem durch die Instrumente deutscher Außenpolitik und die jeweilige „Arbeitsteilung“ zwischen „Praktikern“ und „Akademikern“ ermöglicht werden, erhoffen wir uns ein gleichermaßen facettenreiches wie erfahrungsgesättigtes Bild der Diskussion und die Praxis (,neuer“) deutscher Außenpolitik zu entwerfen. Vor allem eines erhoffen wir uns jedoch von diesem Sammelband: dass er ein weiterer Impuls ist für die (notwendigerweise) nicht endende gesellschaftliche Debatte um außenpolitische Ziele und Inhalte.

Danksagung Für ihre Unterstützung bei Recherchen, Übersetzungen und bei der Erstellung des Gesamtmanuskripts danken wir Daniel Fehrmann und Carina Berg.

\section{Literatur}

Allemann, F. R. (1956). Bonn ist nicht Weimar. Köln: Kiepenheuer \& Witsch.

Bender, P. (1986). Neue Ostpolitik. Vom Mauerbau bis zum Moskauer Vertrag. München: Deutscher Taschenbuchverlag.

Bittner, J., \& Nass, M. (2014). Kurs auf die Welt. Die ZEIT, 06. Februar 2014. http://www.zeit.de/2014/07/ deutsche-aussenpolitik-sicherheitskonferenz/komplettansicht. Zugegriffen: 10. Feb. 2014.

Bredow, W., \& Jäger, T. (1993). Neue deutsche Außenpolitik. Opladen: Nationale Interessen im internationalen System.

Eberwein, W. -D., \& Kaiser, K. (Hrsg.) (1998). Deutschlands neue Außenpolitik. Band 4: Institutionen und Ressourcen. München: R. Oldenbourg Verlag.

Gauck, J. (2014). Rede des Bundespräsidenten zur Eröffnung der 50. Münchner Sicherheitskonferenz am 31. Januar 2014. http://www.bundespraesident.de/SharedDocs/Reden/DE/Joachim-Gauck/Reden/ 2014/01/140131-Muenchner-Sicherheitskonferenz.html;jsessionid=2114CA47EE295D395EBE289 18435B59E.2_cid388?nn=1891550. Zugegriffen: 3. Feb. 2014. 
Haftendorn, H. (2001). Deutsche Außenpolitik zwischen Selbstbeschränkung und Selbstbehauptung, 1945-2000. Stuttgart.

Hellmann, G. (1999). Deutschlands Kraft und Europas Vertrauen oder: Die Selbstbewussten, die Befangenen und die Betroffenen der neuen deutschen Außenpolitik. In C. Lammers \& L. Schrader (Hrsg.), Neue deutsche Außen- und Sicherheitspolitik? Eine friedenswissenschaftliche Bilanz zwei Jahre nach dem Regierungswechsel (AFK-Jahrestagung 1999, Bd. 27 der AFK-Schriftenreihe, S. 42-77). Baden-Baden: Nomos Verlagsgesellschaft.

Hellmann, G. (3. Januar 1997). Jenseits von „Normalisierung“ und „Militarisierung“: Zur Standortdebatte über die neue deutsche Außenpolitik. Aus Politik und Zeitgeschichte, Nr. B1-2, 24-33.

Jäger, T., Höse, A., \& Oppermann, K. (Hrsg.) (2011). Deutsche Außenpolitik. 2. erw. Auflage. Wiesbaden: VS Verlag für Sozialwissenschaften.

Kaiser, K., \& Maull, H. (Hrsg.) (1994). Deutschlands neue Außenpolitik. Band 1: Grundlagen. München: R. Oldenbourg Verlag.

Kaiser, K., \& Maull, H. (Hrsg.) (1995). Deutschlands neue Außenpolitik. Band 2: Herausforderungen. München: R. Oldenbourg Verlag.

Kaiser, K., \& Krause, J. (Hrsg.) (1996). Deutschlands neue Außenpolitik. Band 3: Interessen und Strategien. München: R. Oldenbourg Verlag.

Maull, H. W. (1992). Zivilmacht Bundesrepublik Deutschland: Vierzehn Thesen für eine neue deutsche Außenpolitik. Europa-Archiv, 47(10), 269-278.

Rath, N. (1984). Neu, das Neue. In J. Ritter (Hrsg.), Historisches Wörterbuch der Philosophie (Sp. 728, Bd. 6: Mo-O). Basel: Schwabe AG.

Rinke, A. (2014). Raus ins Rampenlicht. Die Genese der „,neuen deutschen Außenpolitik“. Internationale Politik, Nr. 4 (Juli/August 2014), 8-13.

Risse, T. (2004). Kontinuität durch Wandel: Eine „neue“ deutsche Außenpolitik? Aus Politik und Zeitgeschichte, B11, 24-31.

Schmidt, S., Hellmann, G., \& Wolf, R. (Hrsg.) (2007). Handbuch zur deutschen Außenpolitik. Wiesbaden: VS Verlag für Sozialwissenschaften.

Schöllgen, G. (2003). Der Auftritt. Deutschlands Rückkehr auf die Weltbühne. München: Propyläen Verlag.

Steinmeier, F.-W. (2014a). Rede von Außenminister Frank-Walter Steinmeier anlässlich der 50. Münchner Sicherheitskonferenz, 1.2.2014. http://www.auswaertiges-amt.de/sid_0EEB43D1066AE45F2A36C DB5B6145357/DE/Infoservice/Presse/Reden/2014/140201-BM_M\%C3\%BCSiKo.html. Zugegriffen: 5. Feb. 2014.

Steinmeier, F.-W. (2014b). Rede von Außenminister Frank-Walter Steinmeier anlässlich der Eröffnung der Konferenz „Review 2014 - Außenpolitik Weiter Denken“ am 20. Mai 2014 in Berlin. http://www. auswaertiges-amt.de/DE/Infoservice/Presse/Reden/2014/140520-BM_Review2014.html. Zugegriffen: 28. Mai 2014.

Steinmeier, F.-W. (2013). Rede von Außenminister Frank-Walter Steinmeier bei der Amtsübergabe im Auswärtigen Amt am 17. Dezember 2013. http://www.auswaertiges-amt.de/DE/Infoservice/Presse/ Reden/2013/131217-BM_Antrittsrede.html. Zugegriffen: 9. Jan. 2014. 